\title{
Potency Biomarker Effect of Endothelial Microparticles (EMPs) for Early Prediction of Cardiovascular Risk in Shift Worker Nurses
}

Ike Rahmawaty Alie, MSc, ${ }^{1}$ Hananto Andriantoro, PhD, ${ }^{2}$ Ina S Timan, $\mathrm{PhD},{ }^{3}$ Astrid Widajati Sulistomo, PhD, ${ }^{4}$ Ermita Isfandiary Illyas, $\mathrm{PhD}^{5}$ and Muchtaruddin Mansyur, $\mathrm{PhD}^{4}$

\author{
${ }^{1}$ Doctoral Program of Universitas Indonesia \\ ${ }^{2}$ Department of Cardiology and Vascular Faculty of Medicine, Universitas Indonesia \\ ${ }^{3}$ Department of Pathology Clinic, Faculty of Medicine, Universitas Indonesia \\ ${ }^{4}$ Department of Community Medicine, Faculty of Medicine, Universitas Indonesia \\ ${ }^{5}$ Department of Physiology, Faculty of Medicine, Universitas Indonesia
}

\begin{abstract}
Objectives. Shift work results in changing worker's behavior, food, and sleep patterns, which can cause circadian rhythm disturbance, which is a cardiovascular risk. Until now, a biomarker of early prediction of cardiovascular risk on shift workers is still not developed. This study aimed to assess the cardiovascular risk of shift worker nurses by detecting endothelial microparticles (EMPs).
\end{abstract}

Methods. This longitudinal study compared six shift nurses and five non-shift nurses by measuring the EMPs using antigen $\mathrm{CD} 31^{+}$flow cytometry. All met the inclusion criteria consisting of 28 blood samples followed in one week shift.

Results. EMPs among non-shift nurses were below $200 \mu \mathrm{L}$. However, shift nurses' EMPs increased above $200 \mu \mathrm{L}$ with Man-Whitney $U \mathrm{p}=0.000$ on days 4 and 7 following a one shift per week schedule.

Conclusion. There was an increase in shift workers' endothelial microparticles (EMP) which was a sign of cardiovascular risk.

Key Words: cardiovascular risk, CD31+, endothelial microparticles (EMPs), nurse, shift work

\section{INTRODUCTION}

Paper presented in the $2^{\text {nd }}$ International Conference on Mining and Energy OSH in Conjunction with the $13^{\text {th }}$ Indonesian Occupational Medicine updates on October 7, 2019, at the Sanur, Bali, Indonesia.

Corresponding author: Ike Rahmawaty Alie, MSc

Doctoral Program of Universitas Indonesia

Universitas Indonesia

New Campus UI Depok, West Java - 16424, Indonesia

Email: ikewaty21@gmail.com
As a service provider, hospitals must provide uninterrupted service, thus the need for seamless shift work. Shift work is defined as work time outside the normal working hours, either rotating or permanent, for 24 hours. ${ }^{1,2}$

Shift work is primarily divided into three shifts: morning, afternoon, and night shifts. Shift work can reduce work ability, increase work errors, inhibit social and family relationships, and increase health risks. ${ }^{3}$

About 8.6 million Americans who do shift work are associated with an increased risk of obesity, diabetes, and cardiovascular disease due to a mismatch of circadian rhythms. ${ }^{4,5}$ Studies reported that nurses are predisposed to have high BMI and even obesity attributed to working on night shifts. ${ }^{6}$ The endogenous circadian time system, including the suprachiasmatic nucleus (SCN) in the hypothalamus and peripheral oscillator in vital organs, optimally regulates most physiology and behavior when aligned with 
the sleep or wake cycle. ${ }^{7}$ Circadian rhythm is a biological process regulating the body mechanism on a 24 -hour basis. This mechanism consists of the SCN, the pacemaker center, which regulates the entire body's physiological processes through autonomic and neuroendocrine nervous systems. ${ }^{8}$

Shift work is generally associated with a discrepancy between the time systems of endogenous circadian rhythms and the cycle of behavior, including sleep cycles, wake cycles, and feeding cycles. The SCN regulates leptin rhythms, plasma glucose, glucose tolerance, corticosteroids, and cardiovascular functions through neural or humoral signals to tissues..$^{9}$ The incompatibility in the two systems leads to metabolic and cardiovascular deregulation, which can be detrimental to shift workers. ${ }^{10}$

Generally, nurses in hospitals in Indonesia work in three rotating shifts: a 7-hours morning shift (7:00 to 14:00), a 7-hours afternoon shift (14:00-21:00), and a 10-hours night shift (21:00-07:00). The two conventional rotation models used are Continental Rota [2-2-3 (2) (early in the morning, morning, noon, noon, night, night, night, holidays)] and Metropolitan Rota [2-2-2 (2) early in the morning, noon, nights, holidays) $].{ }^{11}$ Working in shifts at night can incredibly disrupt the circadian rhythm. If continued, it can translate to a cardiovascular risk by disrupting the endothelial function. Therefore, this study aimed to detect the endothelial microparticles (EMPs) using antigen $\mathrm{CD} 31^{+}$of shift workers by flow cytometry.

\section{MATERIAL AND METHODS}

The was a longitudinal study with purposive sampling taking nurses who worked in the hospital. The inclusion criteria were female nurses 25-30 years old with BMI less than $25 \mathrm{~kg} / \mathrm{m}^{2}$, number of years in hospital $>5$ year, and no preexisting cardiovascular disease. The study population was nurses at the in-patient care and policlinic in Al-Ihsan Bandung, West Java, Indonesia. Two groups of eleven nurses consisted of six shift and five non-shift nurses that were twenty-eight blood samples were analyzed. The ethical clearance for the study was approved by the Research Ethics Committee of the Universitas Indonesia (No. 19121964).

The nurses' work shifts, morning, noon, and night shifts, including holidays, were followed. Shift workers' blood samples were taken three times: on the day shift, after the night shift, and after a holiday. Only two blood samples were drawn from non-shift workers: the first and last days of the week. The samples were taken in the morning between 8 and $10 \mathrm{AM}$ to avoid the destruction of blood samples. In addition, the subjects were requested to fast 6 hours before the blood collection.

Three $\mathrm{mL}$ blood samples were collected in Vacutainer ${ }^{\mathrm{TM}}$ Citrate tubes that were centrifuged three times before EMP assessment. The EMPs were detected with flow cytometry antigen-antibody reaction using a specific marker of CD31+. Two $\mathrm{mL}$ of the blood sample was collected from each citrated tube after discharging $1 \mathrm{~mL}$ of the fresh blood. The first centrifuge was done at the speed of 1500 for 15 minutes at room temperature to separate the plasma. The supernatant was removed. Second centrifugation was at the rate of 14000 for 2 minutes at room temperature. The supernatant was removed to collect platelet-poor plasma (PPP). The samples were then frozen, transported, and stored with the tube at $-20^{\circ} \mathrm{C}$ until the following process. The last centrifuge was done at $4000 \mathrm{rpm}$ at $4^{\circ} \mathrm{C}$ for 20 minutes. Finally, a $100 \mu \mathrm{L}$ aliquot was taken for staining with $\mathrm{CD} 31^{+}$and then stored at $-80^{\circ} \mathrm{C}$ for further analysis. Reagents for MPs (microparticles), including FITCAnnexin V (Catalogue number 640906), were obtained from Bioscience. Aliquot samples of $10 \mu \mathrm{L}$ were stained and added to the bead-containing Truecount BD (Catalogue 340440 BD). Annexin buffer and double filter by a 0.22 $1 \mathrm{~m}$ filter was added to the tube to make a total volume and analyzed on the BD Accuri C6 Cytometry (BD Biosciences).

An initial microparticle size gate was set with $1.0 \mu \mathrm{m}$ and $3.0 \mu \mathrm{m}$ calibration particles (Spherotech, Chicago) to detect the microparticles by flow cytometry. We defined the microparticles as particles that would be less than $1.0 \mu \mathrm{m}$. The microparticles with positive staining for annexin and surface markers $\left(\mathrm{CD} 31^{+}\right)$separated the true events from the background noise and unspecific binding of antibodies to debris. Isotype controls were used as the negative control. Absolute count using true count, which used Truecount ${ }^{\mathrm{TM}}$ tubes, were calculated with the appropriate dot plot values entered into a spreadsheet format formula (\# of events in the quadrant containing cell population)/(\# of events in absolute count bead region) x total \# absolute count beads (49600)/test volume $100 \mu \mathrm{L}$. The blood samples were processed at the Pathology Clinic of Cipto Mangun Kusumo Hospital, Jakarta.

Statistical analysis to compare the data was used. Parametric and independent t-test if the data was normal and non-parametric test if the data was not normal. The CD31+ levels between shift nurses and non-shift nurses would be considered statistically significant if the $p$-value was $<0.05$ ( 2 tails).

\section{RESULTS}

Figure 1 describes the differences of the three samples Dn (diamond), Ds (square), and Nd (triangle), representing nurses who worked on shifts. St (cross) represents nurses who were non-shift workers.

It can be concluded that non-shift nurses have a lowlevel CD $31^{+}$compared with nurses who were shift workers. The increase in the levels of CD $31^{+}$in non-shift nurse was not drastic (74 to $\left.111 \mu \mathrm{L} \mathrm{CD} 31^{+}\right)$compared with nurses who worked on three shifts (Dn, from 421 to 268 to 1662 $\mu \mathrm{L}$, Ds, from 380 to 105 to $466 \mu \mathrm{L} \mathrm{CD} 31^{+}$, whether in $\mathrm{Nd}$ from 420 to 240 to $\left.432 \mu \mathrm{L} \mathrm{CD} 31^{+}\right)$. The $\mathrm{CD} 31^{+}$values in non-shift nurses $(\mathrm{St})$ were relatively stable and below 


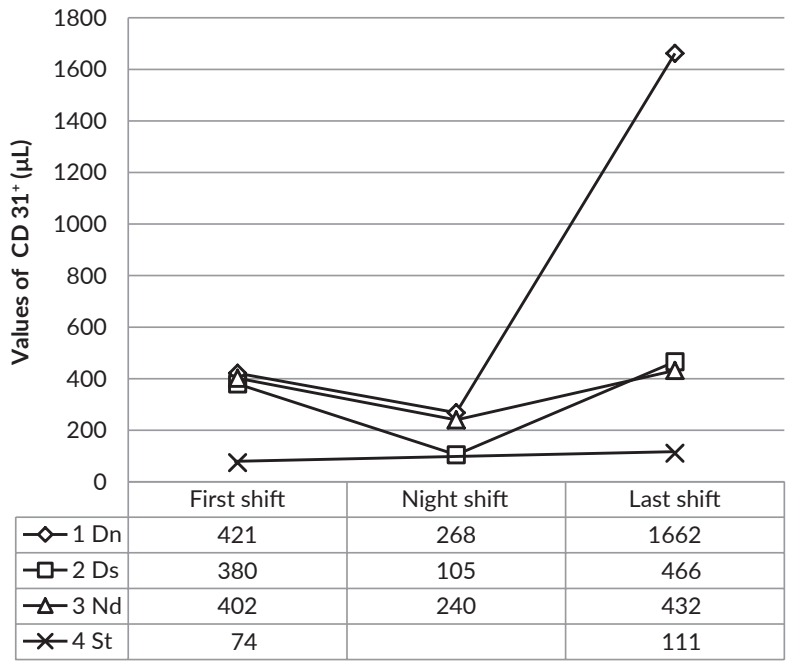

$\mathrm{Dn}, \mathrm{Ds}, \mathrm{Nd}=$ shift worker nurse $\mathrm{St}=$ non-shift nurse

Figure 1. The comparison between mean $\mathrm{CD} 31^{+}$values of first shift, night shift, and last shift among the shift work nurses and non-shift work nurses.

Table 1. Comparison mean of shift and non-shift worker nurse

\begin{tabular}{lcc}
\multicolumn{1}{c}{ Samples } & Mean of EMPs $(\mu \mathrm{L})$ & $\mathbf{p}$ \\
\hline Shift Worker Nurse $(n=18)$ & $427.11(165-1662)$ & 0.000 \\
Non-shift Worker Nurse $(n=10)$ & $115.7 \pm 42.8$ & \\
\hline
\end{tabular}

$p$ significant if $\leq 0.05$

$200 \mu 1$ than shift nurses (Dn, Ds, and Nd), which were higher than $200 \mu \mathrm{L}$.

Table 1 shows the average of the two samples' CD $31^{+}$ taken from non-shift nurses was $115,7 \mu \mathrm{L}$ compared with shift workers' three times of $427,11 \mu \mathrm{L}$. After comparing these with Mann-Whitney U, a significant difference $\mathrm{p}=$

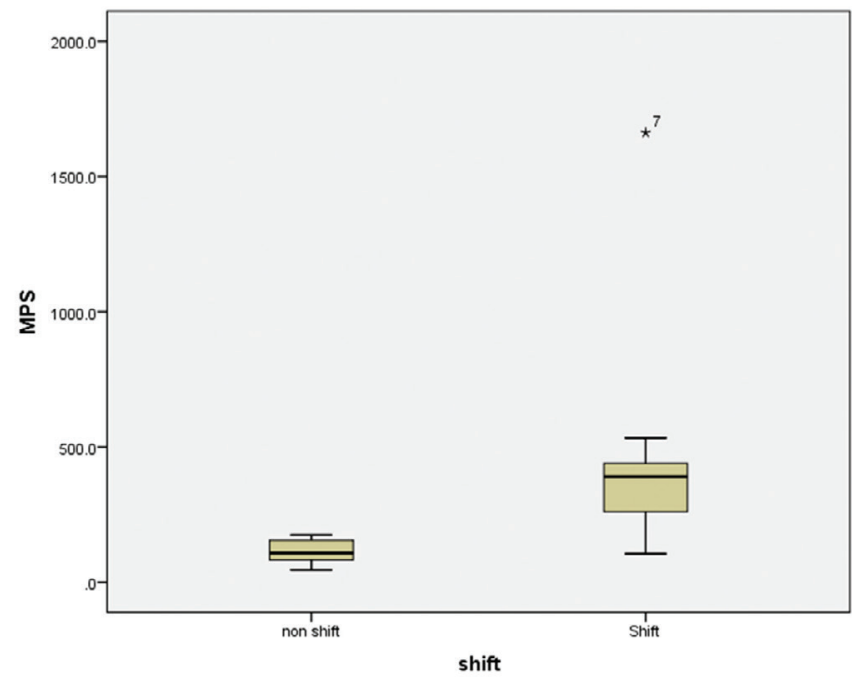

Figure 2. Box plot comparison of microparticles $\left(C D 31^{+}\right)$between shift and non-shift worker nurses.

0.000 (using non-parametric Mann Whitney-U because the data not normally distributed). Figure 2 above is a box plot comparison of microparticles $\left(\mathrm{CD} 31^{+}\right)$between shift and non-shift worker nurses.

Figure 3 shows the shift day of two nurse's samples, Dn (diamond) and Ds (square), to detect endothelial microparticles $\mathrm{CD} 31^{+}$after following a one-week shift schedule. From the picture above, we found that there were two peak curves on days 4 and 7 . First, at night and holidays, CD $31^{+}$increased. Second, based on the CD $31^{+}$ total average, the peak was on days 4 and 7 .

Figure 4 shows one sample's CD31+PE measuring by using zona R4 from the formula by calculating (events in area $\mathrm{CD} 31^{+}(\mathrm{R} 4)$ : event in $\left.\mathrm{R} 2\right) \mathrm{x}$ beads in true count (49600) / volume sample $\mu \mathrm{L}$ ).

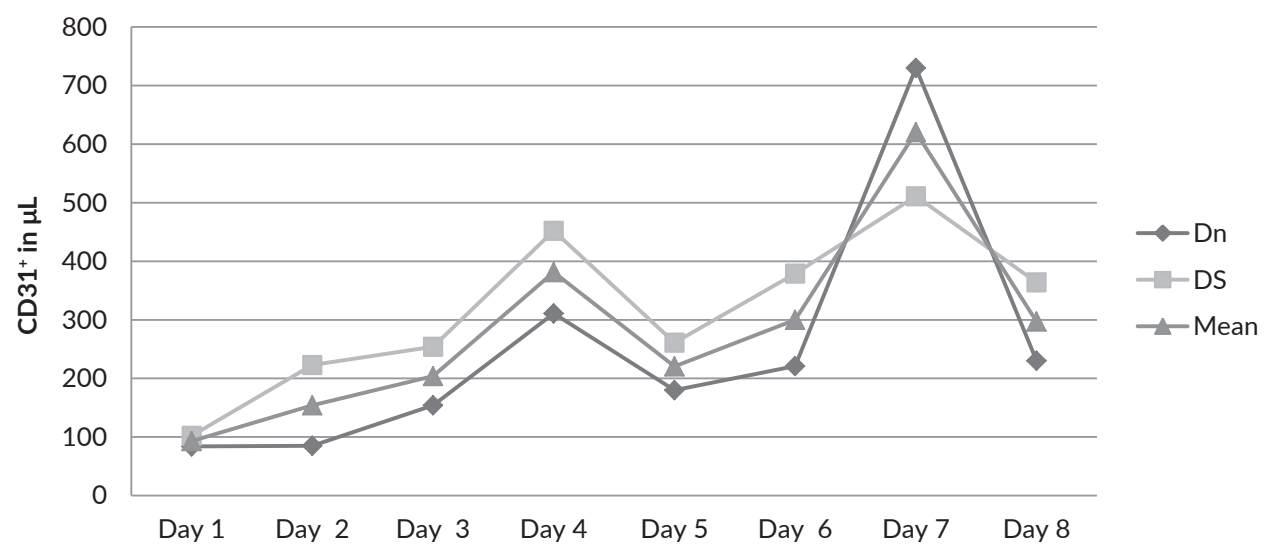

\begin{tabular}{cccccccccc} 
Day & $\mathbf{1}$ & $\mathbf{2}$ & $\mathbf{3}$ & $\mathbf{4}$ & $\mathbf{5}$ & $\mathbf{6}$ & $\mathbf{7}$ & $\mathbf{8}$ \\
Dn & Noon & Morning & Morning & Night & Night & Holiday & Holiday & Noon \\
\hline Ds & Morning & Night & Night & Holiday & Holiday & Noon & Noon & Holiday \\
\hline
\end{tabular}

Figure 3. The comparison between $\mathrm{CD} 31^{+}$values one following week shift among the shift work nurses and non-shift work nurses. 

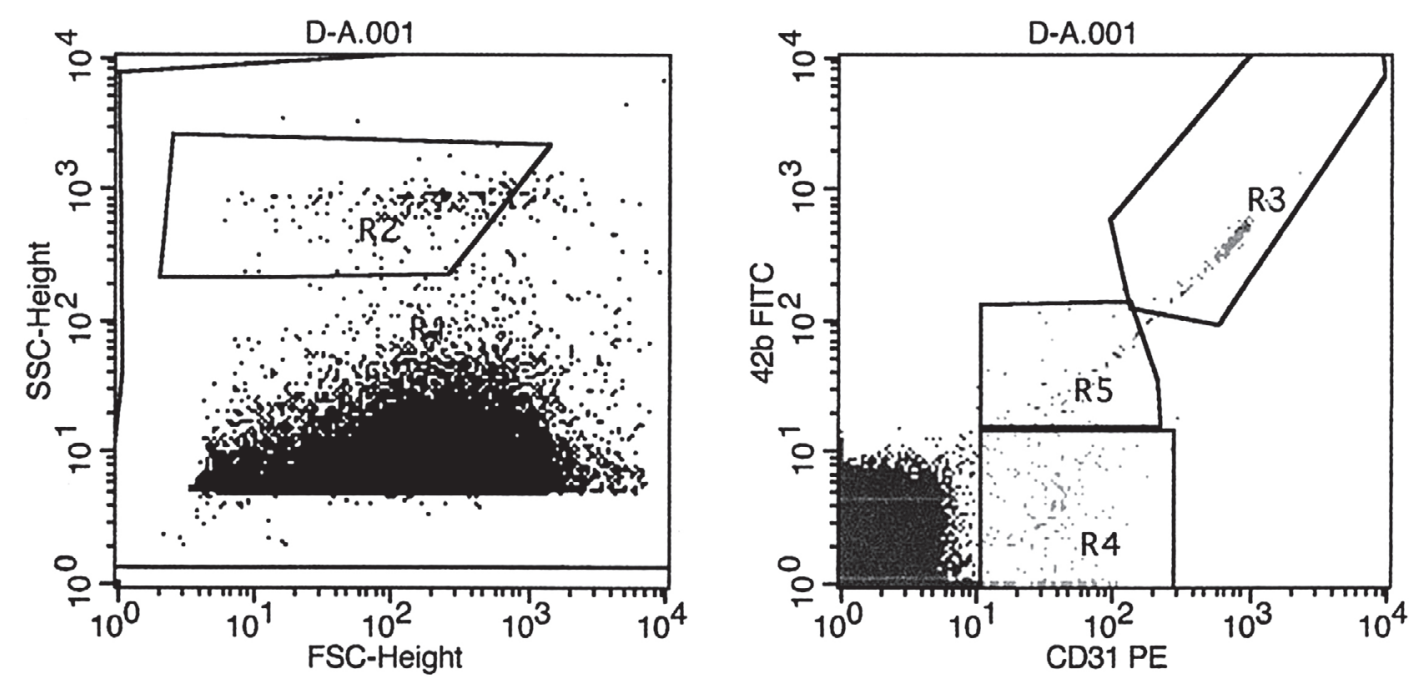

Figure 4. Zone measuring CD31+ in spreadsheet formula.

\section{DISCUSSION}

This study showed EMPs among non-shift nurses are not drastically increased and below $200 \mu \mathrm{L}$, whether in shift nurse drastically increased above $200 \mu \mathrm{L}$ after comparing the mean of two groups (shift worker vs. non-shift workers nurses) Mann Whitney-U $p=0.000$, there statistically significantly different. However, they increased EMPs, especially on day four and day seven, by following one shift working week. This indicated an increase of microparticles by detecting antigen $\mathrm{CD} 31^{+}$on shift workers compared with the non-shift worker, which was a sign of cardiovascular risk.

Changes in the workers' circadian rhythms with the shift rotation method, mostly at night shifts, can affect sleep quality, cortisol, melatonin, and body temperature, increasing the risk of hypertension and other cardiovascular disorders. ${ }^{12}$ Nurses are one of many workers who work in shifts. Working on alternate days such as morning-morningnoon-noon-night-night could change the circadian rhythm.

Changes in the circadian clocks may influence health or disease, which translates to circadian rhythm disruption or sleep deprivation, which is harmful to the cardiovascular system. We found in this study a significant decrease in nitric oxide (NO) in a group of healthy female nurses after three sequential night shifts. This could a sign of impaired endothelial function or endothelial dysfunction. ${ }^{13}$

Endothelial dysfunction is defined as an imbalance between coagulation and thrombosis factors, loss of vasodilatation response-ability in endothelium-dependent stimuli, loss of bioavailability in $\mathrm{NO}$, increased vasoconstriction agents, and disruption in inflammatory regulation, and cell growth in the vascular wall, these are predispositions for atherosclerosis lesions. ${ }^{14}$ One method to predict endothelial dysfunction is to derive the circulating microparticles, especially EMPs, that affect the endothelial nitric oxide synthesis implying their potential role for endothelial autoregulation and thus reflecting the main endothelial system. ${ }^{15,16}$

Microparticles are small cell vesicles released by almost human cells during cellular stress or cell activation. It thereby has been shown that microparticles released by platelets, leukocytes, and endothelial cells can be found in conditions of endothelial dysfunction. ${ }^{17}$ One biomarker based on antibody against specific surface antigen reaction for microparticles endothelial was $\mathrm{CD} 31^{+} .{ }^{18}$

This study found that shift workers' CD $31^{+}$increased on days 4 and 7 following a one-shift working day. The rise in the microparticles may be attributed to a late release of the endothelial microparticles due to the expressing Tissue factor (TF), which required $\mathrm{NF \kappa B}$ and expression of proinflammatory such as IL-1 and TRAIL/Apo2L which fuelled the release of the microparticles. ${ }^{19,20}$

There was an increase of $\mathrm{CD} 31^{+}$microparticles on shift workers compared with non-shift worker nurses, which detects activation of the endothelial cells, which is a sign of earlier endothelial dysfunction. Therefore, an increase of microparticles as CD31 ${ }^{+}$in shift workers could also increase cardiovascular risk.

\section{Limitations of Study}

Due to the preliminary study, the subject of this study is still limited. Still, in a small number of subjects, we can found that in shift worker nurses increasing drastically of microparticles EMPs $\mathrm{CD} 31^{+}$rather than non-shift worker nurses.

\section{CONCLUSION}

Non-shift workers' CD $31^{+}$did not drastically increase and remained below 200/ $\mu$ L. However, shift workers' CD $31^{+}$drastically increased significantly on days 4 and 7 , following a one-shift workday. 


\section{Acknowledgments}

The authors sincerely acknowledge the hospital board and the nurses of Al Ihsan Hospital, Baleendah, West Java, Indonesia.

\section{Statement of Authorship}

All authors participated in data collection and analysis and approved the final version submitted.

\section{Author Disclosure}

All authors declared no conflicts of interest.

\section{Funding Source}

The principal author and BUDI-DN LPDP Indonesia funded this research.

\section{REFERENCES}

1. Tayyari F, Smith JL. Occupational Ergonomics: Principles and Applications, T.J. Press Ltd, Great Britain; 1997. pp. 350.

2. Sack RL, Blood ML, Lewy AJ. Melatonin Rhythms in night Shift Workers. Sleep. 1992 Oct; 15(5):434-41. doi: 10.1093/sleep/15.5.434.

3. Rea MS, Figueiro MG, Sharkey KM, Carskadon MA Relationship of Morning Cortisol to Circadian Phase and Rising Time in Young Adults with Delayed Sleep Times. Int J Endocrinol V.2012 Oct (22):749460. doi: 10.1155/2012/749460.

4. Gu JK, Charles LE, Bang KM, Ma CC, Andrew ME, Violanti JM, Burchfiel CM. Prevalence of Obesity by Occupation Among US Workers. J Occup Environ Med. 2014 May; 56(5): 516-528. doi: 10.1097/JOM.0000000000000133.

5. Luckhaupt SE, Cohen MA, Li J, Calvert GM. Prevalence of Obesity among US Workers and Association with Occupational Factors. Am J Prev.Med 2014 Mar; 46(3):237-48. doi: 10.1016/j. amepre.2013.11.002.

6. Peplonska B, BukowskaA, Sobala W. Association of Rotating Night Shift Work with BMI and Abdominal Obesity among Nurses and Midwives. PLos One 2015 July; 10(7): e0133761. PLoS One. 2015; 10(7): e0133761. 2015 Jul 21. doi: 10.1371/journal.pone.0133761

7. Bushak Lecia. Obesity in the Workplace: Overweight Employees are Less Productive, Need Longer Breaks to Rest. Medical Daily. [Internet] 2014. [cited Jan 2019] available from https://www.medicaldaily.com/ obesity-workplace-overweight-employees-are-less-productive-needlonger-breaks-rest-295160.
8. Martino TA, Sole MJ. Molecular Time: An Often Overlooked Dimension to Cardiovascular Disease. Circulation Research. 2009 Nov; 11(105): 1047-61.

9. Sack RL, Blood ML, Lewy AJ. Melatonin Rhythms in Night Shift Workers. Sleep. 1992; Oct;15(5):434-41. doi: 10.1093/sleep/15.5.434.

10. Depner CM, Stothard ER, Wright KP. Metabolic Consequences of Sleep and Circadian Disorders. Curr Diab Rep. 2014 Juli; 14(7): 507. doi: 10.1007/s11892-014-0507-z.

11. Wickwire EM, Geiger-Brown J, Scharf SM, Drake CL. Shift Work and Shift-Work Sleep Disorder. Chest. 2017 May; 151(5):1156-72. doi: 10.1016/j.chest.2016.12.007.

12. Lieu SJ, Curhan GC, Schernhammer ES, Forman JP. Rotating Night Shift Work and Disparate Hypertension Risk in AfricanAmericans. J Hypertens. 2012 Jan; 30(1):61-6. doi: 10.1097/HJH. 0b013e32834e1ea3.

13. Manfredini R, Portaluppi F. Night Shift and Impaired Endothelial: Circadian Out of Synch May Play a Role. Int J Cardiol. 2012 Jan12;154(1) 94-5. doi: 10.1016/j.ijcard.2011.10.029.

14. Govers R, Ton JR. Cellular Regulation of Endothelial Nitric Oxyde Syntase. Am J Physiol Renal Physiol. 2001 Feb;280(2):F193-206. doi: 10.1152/ajprenal.2001.280.2.F19

15. Berezin A, Zulli A, Kerrigan S, Petrovic D, Kruzliak P. Predictive Role of Circulating Endothelial derived Microparticles in Cardiovascular disease. Clin Biochem. 2015 Jun; 48(9):562-8. doi.org/10.1016/ j.clinbiochem.2015.02.003.

16. Shantsila E. Endothelial Microparticles: A Universal Marker of Vascular Health? J Hum Hypertens. 2009 Nov; 23(5).359-61. doi: 10.1038/jhh.2008.138.

17. Helbing T, Christoph O, Christoph B, Martin M, Philip D. Role of Microparticles in Endothelial Dysfunction and Arterial Hypertension. World J Cardiol. 2014 Nov 26; 6(11): 1135-1139. doi: 10.4330/ wjc.v6.i11.1135.

18. Sansone R, Baaken M, Horn P, Schuler D, Westenfeld R, Amabile N, Kelm M, Heiss C. Endothelial Microparticles and Vascular Parameters in Subjects With and Without Arterial Hypertension and Coronary Artery Disease. Data Brief. 2018 May 8;19:495-500. doi: 10.1016/ j.dib.2018.04.149. PMID: 29900347; PMCID: PMC5997624.

19. Dignat-George F, Boulanger CM. The many faces of endothelial microparticles. Arterioscler Thromb Vasc Biol. 2011 Jan;31(1): 27-33. doi: 10.1161/ATVBAHA.110.218123. PMID: 21160065.

20. Morel O, Jesel L, Freyssinet JM, Toti F. Cellular mechanisms underlying the formation of circulating microparticles. Arterioscler Thromb Vasc Biol. 2011 Jan;31(1):15-26. doi: 10.1161/ATVBAHA. 109.200956. PMID: 21160064.

\section{The Acta Medica Philippina is now accepting limited advertising for its front and back cover (colored), as well as for available spaces in some of its pages, as appropriate. For inquiries and submission of proposals, please email us at actamedicaphilippina.upm@up.edu.ph}

